\title{
Shock Hugoniot of Single Crystal Copper
}

R. Chau, J. Stolken, P. Asoka-Kumar, M. Kumar, N. C. Holmes

September 9, 2009

Journal of Applied Physics 
This document was prepared as an account of work sponsored by an agency of the United States government. Neither the United States government nor Lawrence Livermore National Security, LLC, nor any of their employees makes any warranty, expressed or implied, or assumes any legal liability or responsibility for the accuracy, completeness, or usefulness of any information, apparatus, product, or process disclosed, or represents that its use would not infringe privately owned rights. Reference herein to any specific commercial product, process, or service by trade name, trademark, manufacturer, or otherwise does not necessarily constitute or imply its endorsement, recommendation, or favoring by the United States government or Lawrence Livermore National Security, LLC. The views and opinions of authors expressed herein do not necessarily state or reflect those of the United States government or Lawrence Livermore National Security, LLC, and shall not be used for advertising or product endorsement purposes. 


\title{
Shock Hugoniot of Single Crystal Copper
}

\author{
R. Chau, J. Stölken, P. Asoka-Kumar, M. Kumar, and N. C. Holmes \\ Lawrence Livermore National Laboratory, \\ Livermore, CA 94550
}

(Dated: January 26, 2010)

\begin{abstract}
The shock Hugoniot of single crystal copper is reported for stresses below 66 GPa. Symmetric impact experiments were used to measure the Hugoniots of three different crystal orientations of copper, [100], [110], [111]. The photonic doppler velocimetry (PDV) diagnostic was adapted into a very high precision time of arrival detector for these experiments. The measured Hugoniots along all three crystal directions were nearly identical to the experimental Hugoniot for polycrystalline $\mathrm{Cu}$. The predicted orientation dependence of the Hugoniot from MD calculations was not observed. At the lowest stresses, the sound speed in $\mathrm{Cu}$ was extracted from the PDV data. The measured sound speeds are in agreement with values calculated from the elastic constants for $\mathrm{Cu}$.

PACS numbers:
\end{abstract}




\section{INTRODUCTION}

Material response to a uniaxial shock wave is a well studied area with a long history. From this large body of work, we have a great understanding of the bulk response of many materials over a very broad range of stresses and strains. Despite this knowledge, many processes that take place at the crystal lattice and atomic level are still not understood. The elastic-plastic response of materials, defects and deformation, and crack propagation are just a few examples of open questions. A common assumption in the study of constitutive material response is that the material responds isotropically. The vast majority of shock wave experiments have used polycrystalline materials and the assumption of isotropic response is natural. In monocrystalline materials larger than single crystallite and highly textured materials, the assumption of isotropic response cannot be made. Unlike polycrystalline materials, there is a relative dearth of data on shocked single crystal materials beyond a handful of covalently bonded materials like $\mathrm{LiF}$ [1]. How material response is affected by uniaxial compression along specific crystal orientations has been an open question.

A highly anisotropic material is expected to exhibit strong directional dependence in its shock response. $\mathrm{Cu}$ is an example of a anisotropic solid that has been the subject of some recent interest because of the large anisotropy ratio, $\mathrm{A}=2 \mathrm{c}_{44} /\left(\mathrm{c}_{11}-\mathrm{c}_{12}\right)=3.21$, as calculated from the elastic stiffness constants, $c_{i j}$. Using simple arguments, Bringa and co-workers suggested that a strong crystal orientation dependence would appear in both the elastic and plastic waves in $\mathrm{Cu}$ [4]. Using molecular dynamics (MD) simulations, Bringa et al [4] show a distinct deviation of the Hugoniot from the experimental polycrystalline data of Mitchell et al [6]. The calculated Hugoniot curves (shock velocity, $\mathrm{U}_{s}$, versus mass velocity, $\mathrm{U}_{p}$ ) for the three crystalline directions investigated converge to the polycrystalline curve with increasing $\mathrm{U}_{p}$ but show distinct deviations from the linear fit of the polycrystalline data at lower values of $\mathrm{U}_{p}$. Similar results were reported by Germann et al [5] using a slightly different computational approach. In both cases, the strongest deviations were found in the [110] and [111] directions. However, there are no known experimental observation of crystal orientation dependence in metals.

In this paper, we report the measurement of the shock Hugoniot of single crystal copper along three different crystal orientations. These experiments were performed in the range of stresses between 9 and $67 \mathrm{GPa}$ that corresponds to the regime predicted by the two 
simulation studies to exhibit strong orientation dependence. The upper end of that stress range was chosen to overlap the lowest stresses measured on polycrystalline $\mathrm{Cu}[6]$.

\section{EXPERIMENT}

The reported Hugoniot measurements are based on the method of Mitchell and Nellis [2, 3]. In this method, a $\mathrm{Cu}$ impactor is launched at a target consisting of a two-plane stepped "top hat" made from the same $\mathrm{Cu}$ as the impactor. The impactor and "tophat" target pair for each experiment were made from the same orientation of single crystal copper, ex. [100] impactor and [100] "tophat". Thus, the longitudinal orientation of the impactor and target are matched. However, no attempt was made to match the orientation in the transverse direction. Since this is a symmetric impact experiment, the particle velocity is determined directly from the impactor velocity, $\mathrm{U}_{p}=\mathrm{U}_{I} / 2$. The shock velocity is determined by the shock transit time between the two planes of the top hat target and the distance between the target planes. For direct impact experiments that produce steady waves, $\mathrm{U}_{s}=\Delta \mathrm{x} / \Delta \mathrm{t}$. In the original work of Mitchell and Nellis [2, 3], anodized shorting pins and piezoelectric pins were used as time-of-arrival detectors. For the range of stresses of interest in this study, the shock pressure is too low in the anodized pins for a closure that is both reliable and with a sub-nanosecond rise time. The piezoelectric pins do not have a sufficiently fast rise time to give an accurate determination of the shock transit time. To measure the shock transit time in the $\mathrm{Cu}$ specimen, we adapted the heterodyne velocimetry technique [7] into a high fidelity arrival time detector. The high bandwidth detectors and digitizers used in the heterodyne velocimetry system gives temporal resolution down to $100 \mathrm{ps}$. This is a significant improvement over the temporal resolution of the best trigger pins.

[100], [110], and [111]-oriented, single-crystal copper specimens of radii $8.5 \mathrm{~mm}$ (top plate) and $12.2 \mathrm{~mm}$ (bottom plate) are machined flat and parallel to $2 \mu \mathrm{m}$. The nominal thickness of the bottom plate was $2 \mathrm{~mm}$ while the nominal thickness of the top plate was 1.5 $\mathrm{mm}$. One side (non-impact) received a thin $(0.5 \mu \mathrm{m})$ gold coating to obtain optimum light return into the heterodyne probe system [7]. They are bonded together using Stycast@ 1266 and combined heights before and after bonding are monitored to ensure that the top-hat geometry does not have any significant errors due to the bond layer. Stack heights after

bonding differed from the combined dimensions of the two plates by $0.5 \mu \mathrm{m}$. Impactors 
for each target assembly were machined from the same single crystal starting material with matching crystal orientation and a nominal radius of $28.6 \mathrm{~mm}$ and thickness $1.5 \mathrm{~mm}$. The initial densities of the projectile and target are measured to within $0.1 \%$ using their weights in air and water with appropriate corrections for the thermal expansion coefficient of the material.

Shock arrival times are recorded around two circular arrays of six detectors spaced $60^{\circ}$ apart as described in Ref. [2]. The inner circle (5.5 mm radius) probed the top plane and the outer circle (10 $\mathrm{mm}$ radius) probed the bottom plane. In addition, the shock arrival time at the center point on the bottom plane also is recorded through a hole machined into the top plate (see Fig. 1). Arrival times are recorded using heterodyne velocimetry [7]. In heterodyne velocimetry, beat signals are generated between the Doppler-shifted light reflected from a moving surface and the original unshifted light. A high speed photo detector converts the optical beat pattern to a corresponding electrical signal. Fig. 2(a) shows the beat pattern recorded from a single detector channel. Each detector channel also contained a fiducial time mark that allows cross comparison of arrival time data from all 13 detector channels. Fig. 2(b) shows an expanded view of the heterodyne signal. Shock arrival time is derived as follows. The beat pattern after the shock arrival time is fitted with a sine function and extended to time values before the shock arrival time. The data points before the shock arrival time is fitted with a polynomial and is extended to time values beyond the shock arrival time. The intersection point of the sine and polynomial fits is assigned as the shock arrival time. Shock arrival time for each channel is then corrected for the relative cable (optical and electrical) length differences and lined up to have identical fiducial time mark. We estimate the overall accuracy of this process to be better than 200 ps.

The shock arrival times are examined for consistency as described in Ref. [3]. In gas gun experiments, the projectile is susceptible to small tilt $\left(\sim 1^{\circ}\right)$ and parabolic distortion [3]. Intersection of a shock wave generated with a tilted projectile and the circular array detectors produces a sinusoidal functional form as shown in Fig. 3. The deviation from the sine functional form can be used to assess the quality of the arrival time data. In most cases, the residuals are within $\pm 200 \mathrm{ps}(<0.1 \%)$, better than the results obtained with conventional electrical pin systems and small compared to the shock transit time between the bottom and top planes (340 - $460 \mathrm{~ns})$. The spot size of the optical probes was typically less than 30 $\mu \mathrm{m}$ which minimizes the effect of projectile tilt on the rise time of the heterodyne signal. 
Average shock arrival time in each plane is derived using the results from the circular array of detectors as described in Ref. [2]. Shock transit time is calculated from these values and a parabolic distortion correction, calculated from the data, is applied to account for the projectile distortion. Shock velocity is calculated using the top plate thickness and shock transit time. Actual measured values of the plate thickness (with an accuracy of \pm 0.5 $\mu \mathrm{m})$ at probe locations are used in these calculations. Projectile velocity before impact is measured using flash X-ray images of the projectile in transit at two locations.

\section{ANALYSIS}

The $\mathrm{U}_{s}-\mathrm{U}_{p}$ data for the three crystal orientations of $\mathrm{Cu}$ are shown in Fig. 4. The single crystal data from this study are plotted against the previous polycrystalline data of Mitchell et al for comparison [6]. The inset shows the current single crystal data in comparison to the polycrystalline data of Mitchell et al. The error bars for the single crystal data are obtained using the error analysis as described in Ref. [2]. For the majority of the data in this study, the resulting error bars are less than the size of the marker in the plots. Several of the data points near $\mathrm{U}_{p} \sim 0.92 \mathrm{~km} / \mathrm{s}$ have larger error bars and more scatter. For two of the points, this was due to one or more lost detector channels. Also, these data points were the first experiments in this study and improvements were made in the target assembly and probe alignment after these initial experiments resulting in less scatter. However, the deviation from the polycrystalline EOS for shots 1411 and 1475 (both [100] orientation) are possibly statistically significant and bear further investigation. The data in Fig. 4 and Fig. 5 are summarized in Table I. For these experiments, the transverse orientation of the $\mathrm{Cu}$ projectile was not matched to that of the target (i.e. the projectile was not keyed to the target). We do not expect the transverse orientational mismatch between projectile and target to change the results although this point may need to be tested at some later date.

The Hugoniot data shown in Fig. 4 and 5 show that the Hugoniots for all three crystal orientations show no dependence on the orientation and are in agreement with the polycrystalline $\mathrm{Cu}$ Hugoniot. The solid line in Fig. 4 is a linear fit to the polycrystalline data, and the single crystal data from this study fall exactly on that line within the error bars of the experiment. One can conclude that the dynamic response of single crystal $\mathrm{Cu}$ is indistinguishable with that of polycrystalline $\mathrm{Cu}$. Our results are consistent with the laser driven 
shock experiments on single crystal $\mathrm{Cu}$ shocked along the (200) axis [8,9] to an equivalent hydrostatic stress of 180 kbar. The laser shock experiments used x-ray diffraction showed compression in all directions consistent with that of hydrostatic compression. The transition time from elastic compression to plastic flow was estimated to be of order 10-100 ps [8]. The fact that our data seems indistinguishable from the polycrystalline data is consistent with a rapid relaxation of $\mathrm{Cu}$ to the hydrostat.

Our experimental results disagree with the predictions of both molecular dynamics simulations studies $[4,5]$. The discrepancy between the experimental results and the MD simulations has several possible origins. The timescale of the simulations is several orders of magnitude shorter than our Hugoniot experiments and short compared to the laser experiments. At very short times, a difference along each crystal axis may exist as predicted by the MD simulations. However, any such crystal orientation dependence would likely be overdriven upon the onset of hydrostatic compression. Second, our $\mathrm{Cu}$ samples are single crystals but contain an initial density of defects. The MD simulations did not include extended defects. Such defects may certainly affect the onset of plastic flow and dynamic response.

The primary goal of this study was the measurement of the Hugoniot in single crystal copper but the use of the heterodyne velocimetry diagnostic for time arrival gave additional data in the form of free surface velocity profiles of copper. We note that these experiments were designed and optimized for Hugoniot measurements and not for velocity profile or free surface spall measurements. At the lowest impact velocities, we observed both the elastic and plastic wave in the velocity profiles. The beat signal in Fig. 6 shows the elastic wave traveling ahead of the shock wave for a symmetric copper [110] impact at a velocity of 0.49 $\mathrm{km} / \mathrm{s}$. The extent of the elastic wave signature prior to the shock breakout depends on the crystal orientation and impactor velocity. From the shock speed obtained using Hugoniot relationship (Fig. 3) and the time of arrival of the elastic and shock wave, it is possible to estimate the longitudinal elastic wave speed. For an impact velocity of $0.49 \mathrm{~km} / \mathrm{s}$, we determined the longitudinal elastic wave speeds from the velocity profiles. The experimental and calculated values are listed in Table II. The experimental longitudinal elastic wave speeds are in excellent agreement with the values calculated from the room temperature elastic stiffness constants [10] and Christoffel's secular equation for wave speeds in an anisotropic elastic solid. 
Shown in Fig. 7 are representative velocity profiles for all three orientations of $\mathrm{Cu}$. The data shown in Fig. 7 are from the upper surface of the $\mathrm{Cu}$ stepped target. For all three crystal orientations, spalling of the $\mathrm{Cu}$ was evident in the velocity profiles upon release. A spall pullback was observed in all our experiments from the top surface of the Cu target. A spall signal was not consistently observed on the lower surface of the $\mathrm{Cu}$ target because in many cases the $\mathrm{Cu}$ was too thin at the lower surface. In the cases where a spall signal was detected on the lower surface, the spall plane was estimated to be very close to the actual free surface. The data shown in Fig. 7 is for all six probes observing at nominally the same surface. Along the [100] direction, the six channels are in fairly close agreement with each other. Deviations upon release between the different channels become apparent along the [111] direction while the [110] direction show very strong differences between the different channels. The [110] direction consistently showed differences among the different channels on each shot while the [111] showed small differences upon release. It is interesting to note that the average spot size of the probes for these experiments is less than $30 \mu \mathrm{m}$. One conjecture is that we are observing microstructural differences between the different probe positions since the probe area is sufficiently small. If we average all of the velocity profiles on a given shot, the average velocity profile is consistent with previously reported $\mathrm{Cu}$ profiles under similar loading conditions $[11,12]$. Some of the observed behavior could be attributed to radial edge releases since the probe placement was not chosen to maintain 1D compression for any appreciable duration of time at the probe locations. There are interesting aspects of

the spall behavior in the single crystal samples we studied. A full and detailed analysis of the velocity profiles is beyond the scope of this current study but suggests some interesting behavior.

\section{CONCLUSION}

In summary, we have measured the Hugoniot of single crystal copper along three different crystal orientations. The measured Hugoniot for all three orientations are in very good agreement with the polycrystalline copper Hugoniot. Our results do not show any crystal orientation dependance of the Hugoniot as predicted by MD simulations [4, 5]. This result is consistent with the experimental results from laser shock experiments that suggest that copper rapidly relaxes to the hydrostat under dynamic loading. The sound velocity of copper 
along the three elastic constants was determined from the velocity of the elastic and plastic waves measured in the lowest stress experiments. The measured sound velocities agree very well with the values calculated from the crystal coefficients for copper. Copper spall was observed in the velocity profiles in all of our experiments. A full analysis is not offered at this time due to uncertainties about radial and edge effects. While no dependence of the Hugoniot was observed in our experiments, the strength of copper may have dependence on orientation and may warrant further study.

\section{ACKNOWLEDGEMENT}

This work was performed under the auspices of the U.S. Department of Energy by Lawrence Livermore National Laboratory in part under Contract W-7405-Eng-48 and in part under Contract DE-AC52-07NA27344.

[1] Y.Gupta, J. Appl. Phys. 48, 5067 (1977).

[2] A. C. Mitchell and W. J. Nellis J. Appl. Phys. 52, 3363(1981).

[3] A. C. Mitchell and W. J. Nellis Rev. Sci. Instrum. 52, 347(1981).

[4] E. M. Bringa, J. U. Cazamias, P. Erhart, J. Stölken, N. Tanushev, B. D. Wirth, R. E. Rudd, and M. J. Caturla J. Appl. Phys. 96, 3793 (2004).

[5] T. C. Germann, B. L. Holian, and P. S. Lomdahl Phys. Rev. Lett. 84, 5351 (2000).

[6] A. C. Mitchell, W. J. Nellis, J. A. Moriarty, R. A. Heinle, N. C. Holmes, R. E. Tipton, and G. W. Repp, J. Appl. Phys. 69, 2981 (1991).

[7] O.T. Strand, D.R. Goosman, C. Martinez, T.L. Whitworth, and W.W. Kuhlow Rev. Sci. Instrum. 77, 08310 (2006).

[8] A. Loverridge-Smith, A. Allen, J. Belak, T. Boehly, A. Hauer, B. Holian, D. Kalantar, G. Kyrala, R. W. Lee, P. Lomdahl, M. A. Meyers, D. Paisley, S. Pollaine, B. Remington, D. C. Swift, S. Weber, and J. S. Wark Phys. Rev. Lett. 86, 2349 (2001).

[9] M. A. Meyers, F. Gregori, B. K. Kad, M. S. Schneider, D. H. Kalantar, B. A. Remington, G. Ravichandran, T. Boehly, J. S. Wark Acta Mater. 51, 1211 (2002).

[10] H. M. Ledbetter and E. R. Naiman , J. Phys. Chem. Ref. Data 3, 897 (1974). 
[11] R. W. Minich, J. U. Cazamias, M. Kumar, and A. J. Schwartz Metall. Mater. Trans. A-Phys. Metall. Mater. Sci. 35A, 2663(2004).

[12] J. N. Johnson, G. T. Gray III, and N. K. Bourne J. Appl. Phys. 86, 4892(1999). 
TABLE I: Experimental Hugoniot data for single crystal copper.

\begin{tabular}{cccccc}
\hline \hline shot no. & Orientation & $\mathrm{U}_{I}(\mathrm{~km} / \mathrm{s})$ & $\mathrm{U}_{s}(\mathrm{~km} / \mathrm{s})$ & relative volume & Longitudinal Stress $(\mathrm{GPa})$ \\
\hline 1389 & 110 & $0.960 \pm 0.00096$ & $4.606 \pm 0.0490$ & $0.8958 \pm 0.0014$ & $19.71 \pm 0.213$ \\
1390 & 100 & $0.950 \pm 0.00095$ & $4.636 \pm 0.0190$ & $0.8975 \pm 0.001$ & $19.67 \pm 0.087$ \\
1391 & 111 & $0.940 \pm 0.00094$ & $4.637 \pm 0.0190$ & $0.8986 \pm 0.001$ & $19.40 \pm 0.085$ \\
1406 & 110 & $0.493 \pm 0.00200$ & $4.386 \pm 0.0036$ & $0.9438 \pm 0.001$ & $9.64 \pm 0.390$ \\
1407 & 100 & $0.490 \pm 0.00049$ & $4.268 \pm 0.0170$ & $0.9426 \pm 0.0009$ & $9.31 \pm 0.040$ \\
1408 & 111 & $0.490 \pm 0.00049$ & $4.282 \pm 0.0170$ & $0.9428 \pm 0.0009$ & $9.37 \pm 0.039$ \\
1411 & 100 & $1.820 \pm 0.00182$ & $5.051 \pm 0.0390$ & $0.8198 \pm 0.0016$ & $41.05 \pm 0.319$ \\
1412 & 110 & $0.940 \pm 0.00094$ & $4.658 \pm 0.0260$ & $0.8991 \pm 0.0011$ & $19.52 \pm 0.111$ \\
1418 & 111 & $1.840 \pm 0.00200$ & $5.261 \pm 0.0420$ & $0.8245 \pm 0.0016$ & $43.23 \pm 0.348$ \\
1474 & 110 & $1.840 \pm 0.00200$ & $5.216 \pm 0.0360$ & $0.8236 \pm 0.0015$ & $42.96 \pm 0.304$ \\
1475 & 100 & $1.824 \pm 0.00200$ & $5.166 \pm 0.0570$ & $0.8235 \pm 0.0015$ & $42.07 \pm 0.294$ \\
3954 & 110 & $1.928 \pm 0.00200$ & $5.450 \pm 0.0260$ & $0.8231 \pm 0.0012$ & $47.03 \pm 0.235$ \\
3955 & 111 & $2.400 \pm 0.00200$ & $5.797 \pm 0.0280$ & $0.7929 \pm 0.0013$ & $62.22 \pm 0.315$ \\
3956 & 100 & $2.508 \pm 0.00200$ & $5.841 \pm 0.0290$ & $0.7852 \pm 0.0013$ & $65.61 \pm 0.343$ \\
\hline \hline
\end{tabular}

TABLE II: Experimental and Calculated Elastic Wave Speeds.

\begin{tabular}{ccc}
\hline \hline Orientation & Experimental Elastic Wave Speed $(\mathrm{km} / \mathrm{s})$ & Calculated Elastic Wave Speed $(\mathrm{km} / \mathrm{s})$ \\
\hline 100 & $4.303 \pm 0.0065$ & 4.357 \\
110 & $4.907 \pm 0.0070$ & 4.962 \\
111 & $5.053 \pm 0.0072$ & 5.148 \\
\hline \hline
\end{tabular}




\section{Impactor}
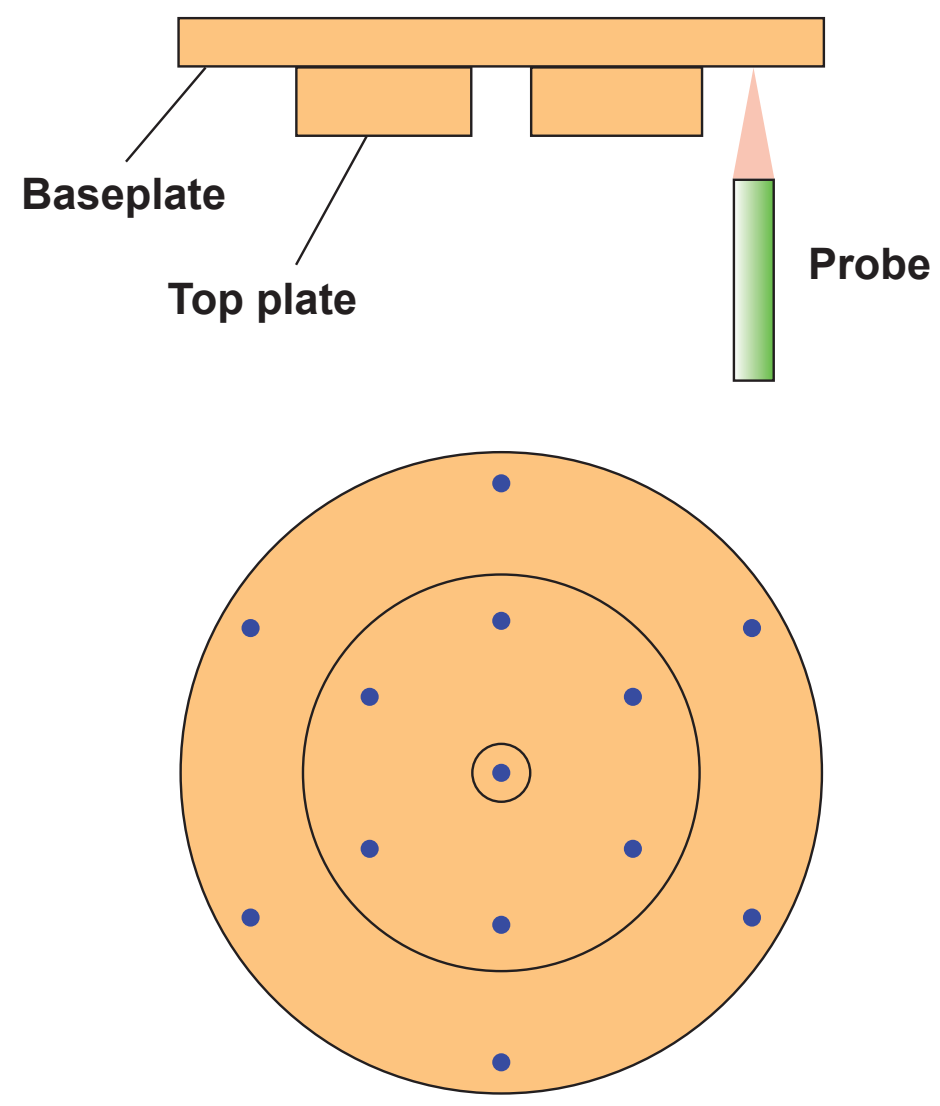

FIG. 1: A diagram of target and probe positions. 

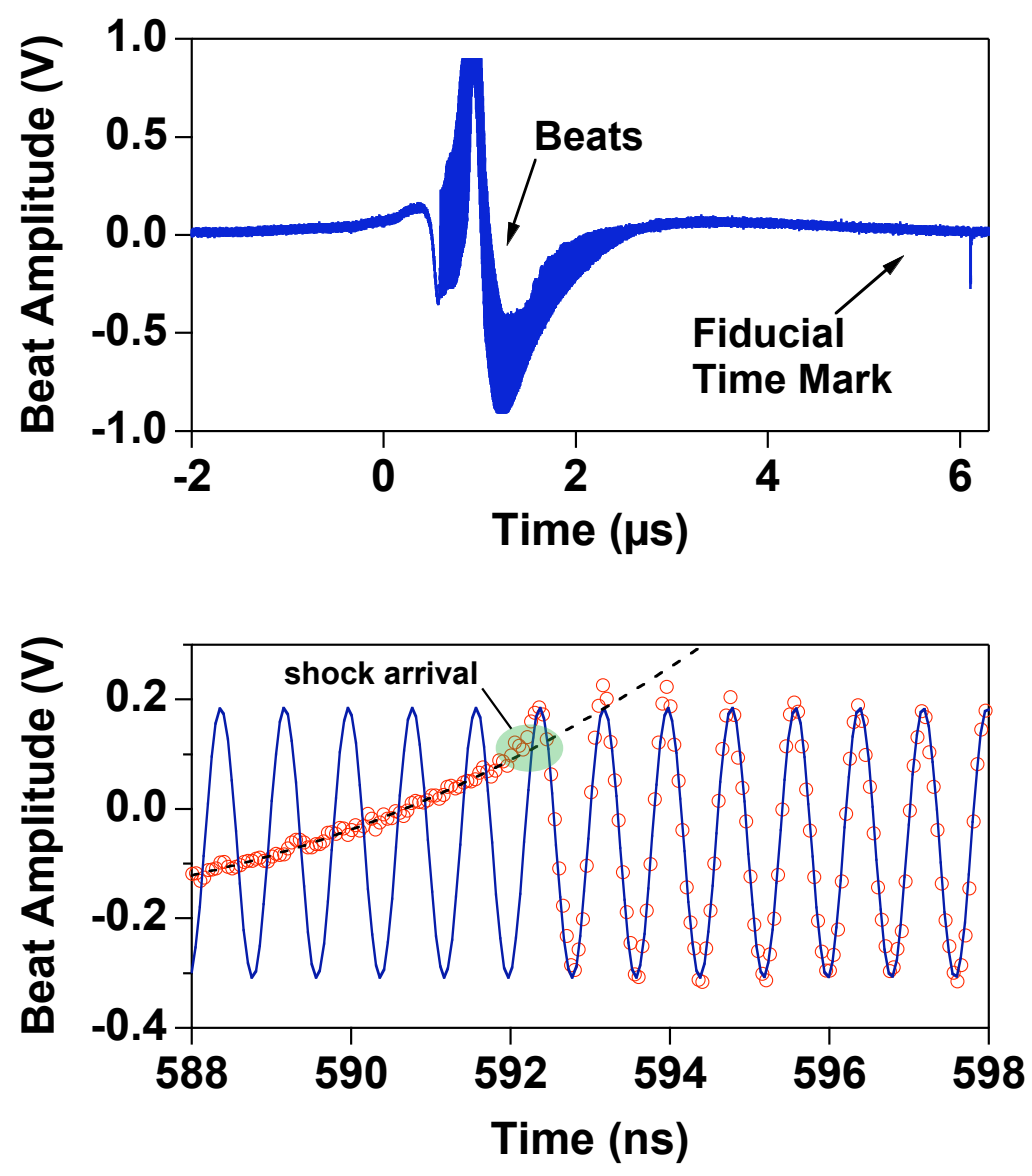

FIG. 2: (a) A typical beat frequency pattern is shown for one channel. The negative peak at late times is the superimposed fiducial timing mark. (b) The expanded view of the beat data is shown. The open circles are the actual data while the smooth line is a sine wave fit to the beat pattern. The dashed line is a polynomial fit to the data before shock arrival. Shock arrival time is determined by the intersection of the sine wave fit and the polynomial fit. 


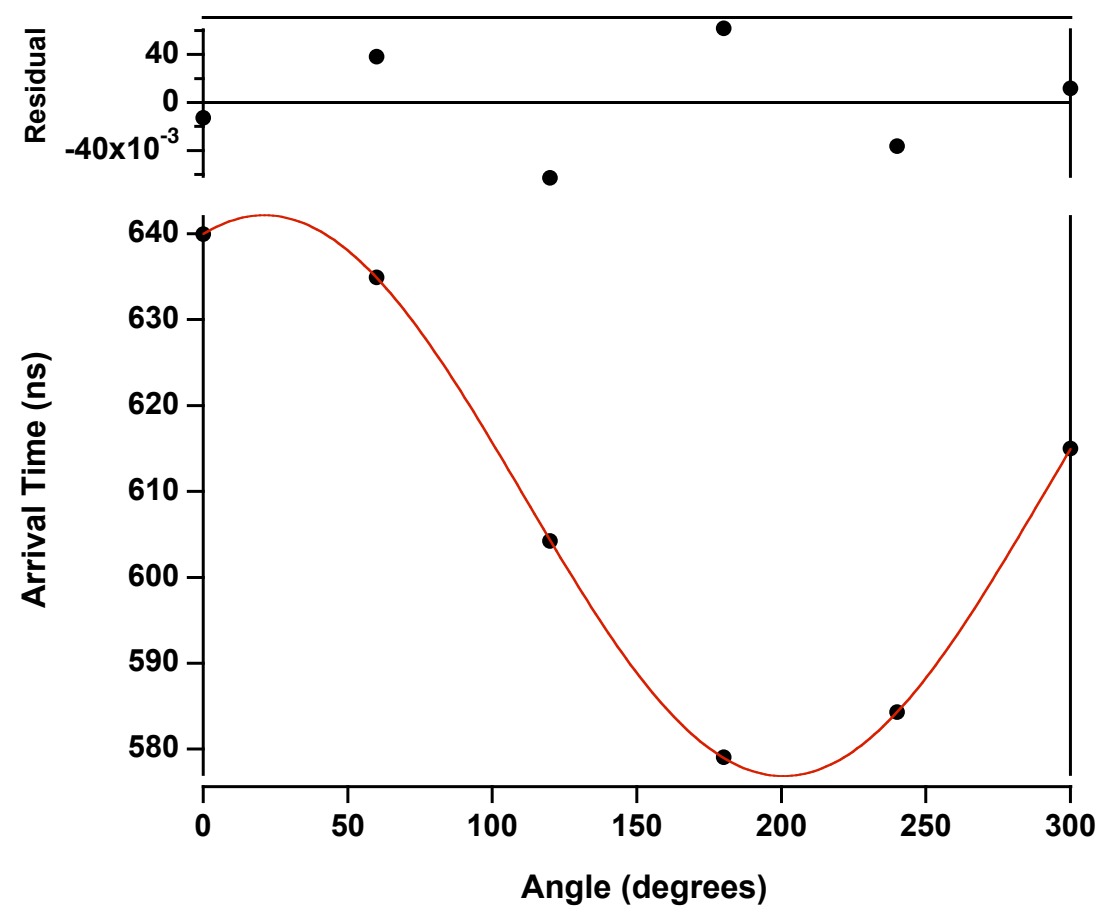

FIG. 3: A fit of the arrival time data for one circle of probes to a sine wave is shown. The residuals for the fit are also shown and give a measure of the quality of the fit. 


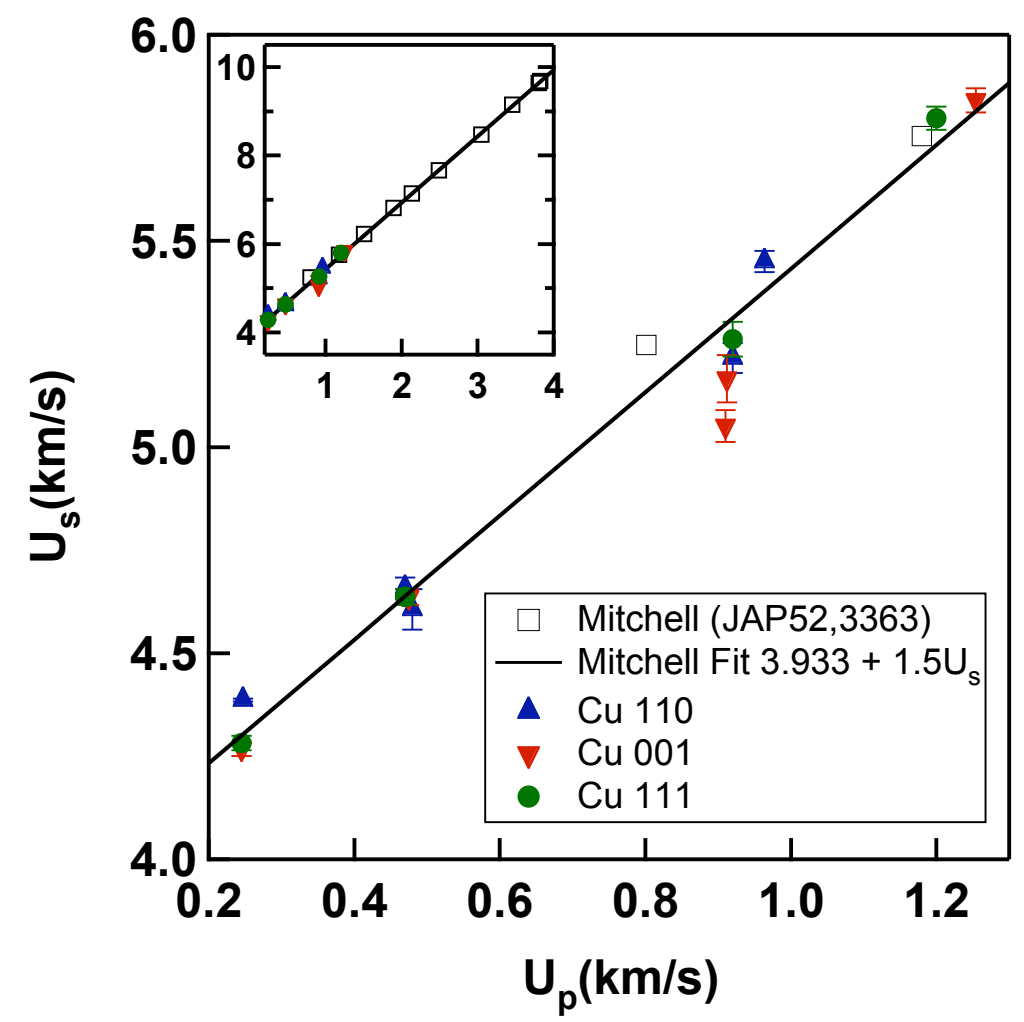

FIG. 4: The Hugoniot of single crystal $\mathrm{Cu}$ is plotted as $\mathrm{U}_{s}$ vs. $\mathrm{U}_{p}$. The open squares are previous data by Mitchell and Nellis on polycrystalline $\mathrm{Cu}$. The solid line is a fit of the Mitchell-Nellis data to a linear $\mathrm{U}_{s}-\mathrm{U}_{p}$ form. The inset shows the polycrystalline data of Mitchell-Nellis in comparison to the single crystal data. 


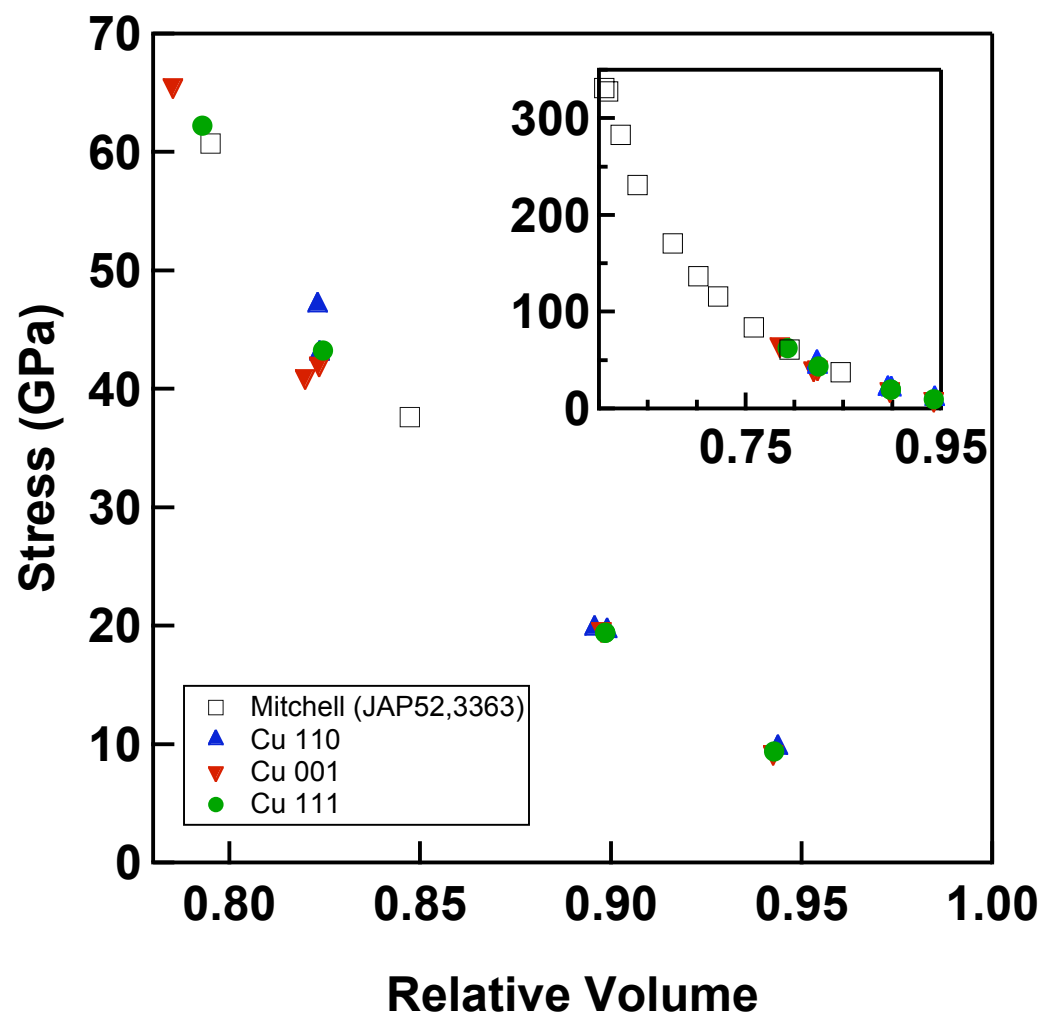

FIG. 5: The EOS of $\mathrm{Cu}$ plotted in $\mathrm{P}-\rho$ space. The inset shows an expanded view including the higher stress polycrystalline data of Mitchell et al. 


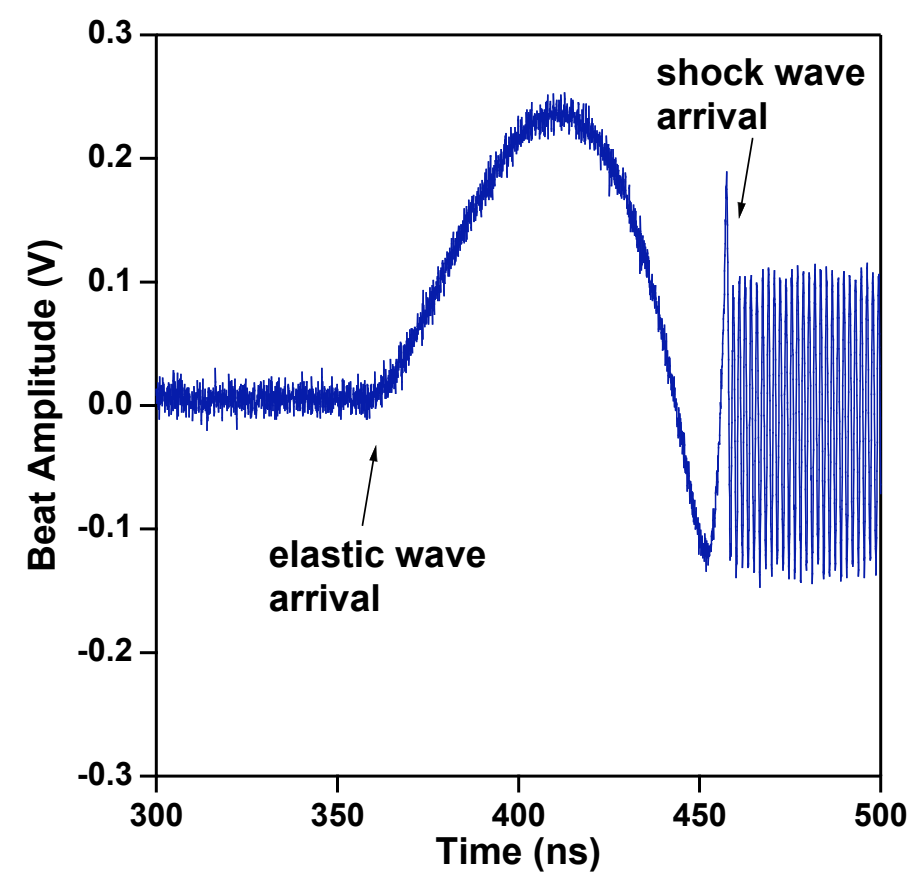

FIG. 6: The beat signal from the symmetric impact of [110] $\mathrm{Cu}$ at an impact velocity of $0.49 \mathrm{~km} / \mathrm{s}$. The elastic wave can be seen traveling ahead of the shock wave. 

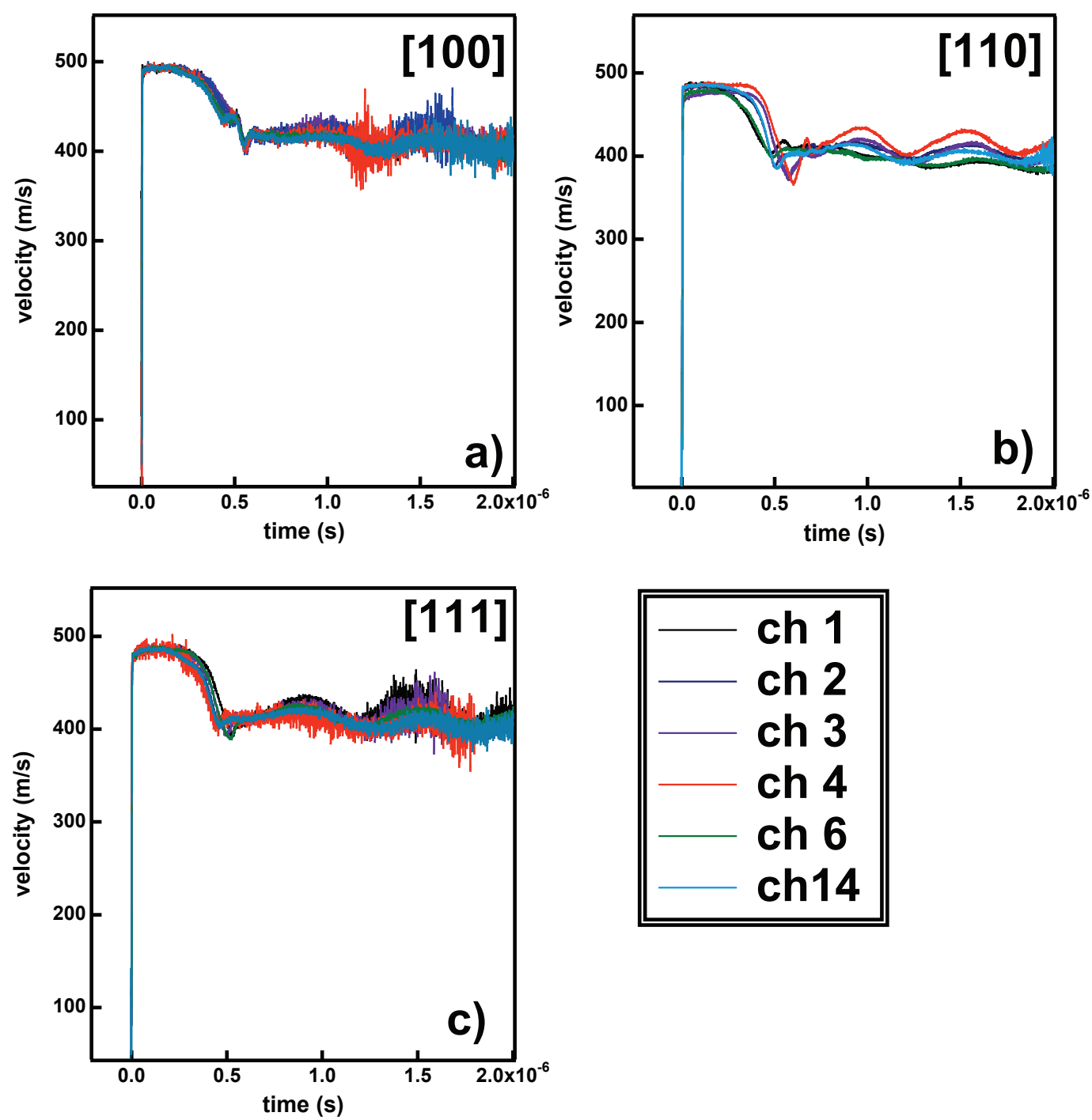

FIG. 7: Representative velocity profiles for a) [100] Cu, b) [110], c) [111]. The velocity profiles are shown from the upper surface of the $\mathrm{Cu}$ target and have been shifted in time to coincide with each other. 